\title{
Electron Cyclotron Wave Propagation, Absorption, and Backscatter Measurements in a Laboratory Plasma
}

\author{
John E. Scharer, Member, IEEE, Owen C. Eldridge, Sheng-Fuh R. Chang, Member,
} IEEE, Yong-Shan Zhang, Michael H. Bettenhausen, and Nguyen T. Lam

\begin{abstract}
The subject of broadband microwave propagation and absorption processes and backscatter from objects immersed in a magnetized, finite plasma column is addressed. The propagation, absorption, and backscatter of electron cyclotron waves from an object in a warm, magnetized plasma are measured and compared with bounded vacuum hot plasma wave propagation, absorption, and ray tracing theory. The nonreciprocal nature of the electron cyclotron wave transmission and absorption in an anisotropic plasma is measured. A homodyne technique which isolates the scattering from a single object in the plasma from the scattering from all other objects in the plasma and the walls of the containment device is developed and utilized. The range of absorption frequencies and nonreciprocity of the transmission signal are shown to be well correlated with wave trajectories in the associated regions of the Clemmow-Mullaly-Allis (CMA) diagram. It is shown by experimental measurement, ray tracing and analysis of a vacuum boundary plasma column that quasiparallel propagation of electron cyclotron waves near resonance is present and that the transverse wavenumber effects on propagation in the cylindrical plasma $\left(\left(f_{p} / f\right)^{2} \geq 6 . f / f_{c e} \simeq 0.9\right)$ are small. An examination of cases in which damping occurs is shown to provide a stronger damping than WKB theory would predict, which is suggested by previous theoretical work. The experimental methods have a powerful potential for further basic plasma physics wave studies.
\end{abstract}

\section{INTRODUCTION}

$\mathbf{E}$ LECTRON cyclotron wave propagation and absorption have been and continue to be an intriguing plasma physics research area. McVey and Scharer [1], [2] examined the theoretical hot plasma collisionless spacial damping rate for a right-hand circularly polarized (RHCP) electromagnetic wave and experimentally verified the dispersion and collisionless electron cyclotron damping rates near and somewhat removed from resonance in a weak magnetic field gradient which satisfied Wentzel-Kramers-Brillouin (WKB) criteria. Pulsed diamagnetic loop measurements by Owens and Scharer [3] verified that the wave damping corresponds to an increased transverse plasma temperature.

Later work by Scharer and Mitzlaff [4], Rice and Scharer [5], and Mett, Lam, and Scharer [6] examined parametric

Manuscript received June 29, 1992; revised February 26, 1992. This work was supported by Air Force Office of Scientific Research Grant 89-0353 and, in part, by the University of Wisconsin Graduate School, Department of Energy Grant FG02-86ER53218, Task III and National Science Foundation Grant ECS-8514978.

The authors are with the University of Wisconsin, Madison, WI 537061687.

IEEE Log Number 9208939 instabilities and temperature elevations occurring near electron cyclotron resonance at higher power levels $(\geq 10 \mathrm{~W})$. Recent work by Booske et al. [7] has examined the electron temperature anisotropy and whistler instability spectra that can be created by sufficiently high power pulsed heating in a magnetic mirror. Stenzel and Urrutia [8] have also experimentally examined force-free, intense electromagnetic pulses which propagate along magnetic field lines as a whistler mode.

In this paper we present new measurements of several related properties of electromagnetic wave propagation in a magnetized plasma. They include broadband network analyzer measurements of antenna coupling, transmission, and reception of plasma wave modes for the RHCP electron cyclotron mode near and far from cyclotron resonance. An additional new experiment involves the measurement of microwave scattering of plasma waves from a moveable modulated probe that is imbedded in the inhomogeneous plasma. This sensitive technique minimizes the effects of the boundaries for basic physics measurements of wave propagation in small laboratory experiments.

These quantitative measurements and the associated analyses extend the regimes of observation for basic plasma physics properties of electron cyclotron waves. The wide band transmission and absorption properties of the wave in an inhomogeneous plasma are measured and analyzed. The antenna transmitting and receiving characteristics are measured and the nonreciprocal property of the intervening plasma region is observed and interpreted. In addition to the network analyzer and homodyne measurement techniques used in this experiment, the work differs in several other respects from earlier work. [1]-[4]. The electron cyclotron resonance produced plasma column of diameter $d=12 \mathrm{~cm}$ is much larger than that produced earlier, and the magnetic beach has steeper gradients $\left.\left(L_{B}=[(1 / B) \mathrm{dB} / \mathrm{dz})\right]^{-1} \gtrsim 18 \mathrm{~cm}\right)$. In addition, larger wave damping decrements near resonance relative to the local parallel wavenumber, $k_{i} / k_{r} \simeq 0.3-0.6$, which do not satisfy WKB criteria are observed.

The paper is organized in the following manner. In Section II, the detailed experimental setup and diagnostics are described. In Section III, results of the network analyzer, backscatter and direct wave measurements are presented. The results are discussed, quantitatively compared with theoretical predictions, and interpreted in Section IV. The results are summarized in Section V. 


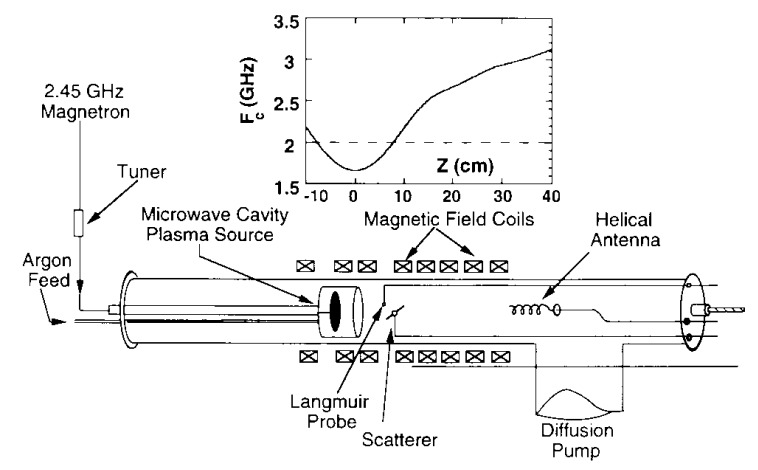

Fig. 1. Schematic of experimental apparatus. The inset shows the cyclotron frequency vs. axial distance $\approx$ for a field coil current $I_{r}=290 \mathrm{~A}$.

\section{EXPERIMENTAL DESCRIPTION}

\section{A. Experimental Configuration}

The experimental layout is shown in Fig. 1. The measurements are carried out for a $12 \mathrm{~cm}$ diameter plasma inside a glass tube of $15.2 \mathrm{~cm}$ inner and $17.2 \mathrm{~cm}$ outer diameter, $200 \mathrm{~cm}$ in length and wrapped with copper screen to provide shielding and a well-defined radiofrequency ( $\mathrm{rf}$ ) boundary condition. A diffusion pump provides a base pressure of $8 \times 10^{-7}$ Torr. Eight water cooled magnetic field coils at currents of $I_{c}=290$ A corresponding to a cyclotron resonance at $z=0$ of 1.66 $\mathrm{GHz}$ are aligned to produce the on-axis axial magnetic field profile shown in Fig. 1. The location of the magnetic field minimum, inferred from a least squares parabolic fit to the data for $-3<z<3 \mathrm{~cm}$, is defined as $z=0$. The central mirror extends from -20 to $20 \mathrm{~cm}$ with a mirror ratio of 1.6 . Most experimental wave data are taken in the $-10<z<40$ $\mathrm{cm}$ region. The microwave cavity plasma source, positioned in the $-40<z<-30 \mathrm{~cm}$ region, is driven by a $2.45 \mathrm{GHz}$ $\mathrm{CW}$ magnetron at power levels from 70 to $200 \mathrm{~W}$. The plasma is created by electron cyclotron resonance heating (ECRH) in the cavity with argon at pressures ranging from $2 \times 10^{-4}$ to $8 \times 10^{-4}$ Torr. The gross absorption is extremely efficient; with $150 \mathrm{~W}$ of microwave power at the source cavity, the intensity of radiation escaping from the ends is less than $1 \mu \mathrm{W} / \mathrm{cm}^{2}$.

A helical launch antenna in the region $40<z<50$ $\mathrm{cm}$, where $f / f_{c e}=0.6$, is driven in the frequency range $1<f<3 \mathrm{GHz}$ by a signal generator or network analyzer. Earlier experiments [1], [2], [5] have verified that this type of antenna primarily launches a right-hand circularly polarized wave with respect to the magnetic field when $f_{p}>f$ as is the case for these experiments.

The plasma density $(n)$ and the electron temperature $(T)$ are measured with small Langmuir probes consisting of 0.24 $\mathrm{cm}$ diameter tantalum dises $1.3 \times 10^{-2} \mathrm{~cm}$ in thickness. The voltage applied to the probe is ramped from $-40 \mathrm{~V}$ to $+40 \mathrm{~V}$ in $200 \mathrm{~ms}$ while the current is monitored, and the characteristic is measured every 2 seconds. The Langmuir probe is moved along the axis from $z=-10 \mathrm{~cm}$ to $z=40$ $\mathrm{cm}$, producing about 450 characteristic curves. The probe characteristic curves are analyzed in real time with a computer.
The small receiving antennas are moved through the same range by the probe drive.

The electron temperature is $3<T_{e}<5 \mathrm{eV}$ while the ion temperature is near room temperature due to the plasma heating method and moderate neutral pressure range. The background plasma on axis density for these measurements is in the range $5 \times 10^{10}<n<3.3 \times 10^{11} \mathrm{~cm}^{-3}$ as determined by Langmuir probe measurements which agree with hot plasma theory [2] density predictions of local on-axis wavelength measurements within $\pm 20 \%$.

\section{B. Data Acquisition and Analysis}

Data acquisition and analysis are performed with a computer and data acquisition board with a sampling rate of 10000 per second. A signal conditioning unit converts the signals from the Langmuir and magnetic probes and laboratory equipment into the $\pm 5 \mathrm{~V}$ range utilizing 12 bits of resolution. This unit also sweeps the Langmuir probes and allows the computer to sweep the spectrum analyzer. Signal conditioning is performed by instrumentation amplifiers. Data is recorded and archived for subsequent analysis.

An indication of the wave transverse electric field is also obtained by connecting a Langmuir probe or dipole antenna in series with a microwave feed-through capacitor to the same apparatus used to measure the wave magnetic field. The Langmuir probe stalks are $6 \mathrm{~cm}$ in length, aligned perpendicular to the magnetic field, and shielded from the plasma with ceramic tubing. The signal is carried from the machine through 0.12 $\mathrm{cm}$ diameter semirigid coaxial cable. In this configuration the probes function as monopole or dipole antennas.

\section{Helical Antenna}

The helical antenna design is based on the assumption that the antenna properties in the plasma can be approximated by treating the plasma as a homogeneous dielectric medium with properties characterized by the wavelength for a right-hand circularly polarized cyclotron wave propagating parallel to the magnetic field. Although this assumption is not fully satisfied in the experiment, it leads to a tractable means for designing a useful antenna. The wavelength is calculated from the local hot plasma dispersion relation for the plasma parameters at the helix location.

The typical electron cyclotron wavelength in the antenna region is $7 \mathrm{~cm}$ under conditions of operation at $2 \mathrm{GHz}$, a plasma density of $1.4 \times 10^{11} \mathrm{~cm}^{-3}$ and a local ratio of wave frequency to electron cyclotron resonance frequency of 0.62 in the helix region. The axial pitch and circumference for the helix were chosen to satisfy the criteria for axial mode operation of the helix as given by Stutzmann and Theile [9]. A circumference of $C=7 \mathrm{~cm}$, a pitch angle $\alpha$ of $13^{\circ}$ and seven $2.2 \mathrm{~cm}$ diameter turns were used for the helical antenna. A $6 \mathrm{~cm}$ diameter copper screen ground plane was placed at the coax to helix transition. The mismatch caused by the $50 \Omega$ coax to helix connection typically caused a $2 \mathrm{~dB}$ reduction in the transmitted signal power. For the backscattering experiments, we used a double stub tuner to match the generator to the helix when immersed in the plasma. 


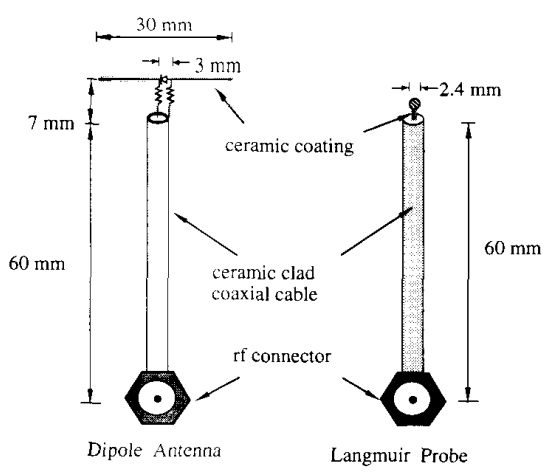

Fig. 2. Dipole antenna and Langmuir probe.

\section{Modulated Dipole Antenna and Homodyne System}

To tag the scatterer and differentiate it from the rest of the experimental configuration we utilize a dipole antenna as shown in Fig. 2. It is made of a single microwave PIN (ptype, intrinsic type, n-type) diode (HP 5082-3042) which has a very small conduction resistance and fast response to ensure optimum modulation. The diode can be switched on and off through two resistors which change the wave scattering crosssection accordingly. The value of the two resistors is selected to achieve full forward biasing and large parallel resistance loading when reverse biased. In our experiment, two $10 \mathrm{k} \Omega$ resistors are used. The amplitude of the modulating signal is a square wave of $20 \mathrm{~V}$ which gives a forward bias current of $1 \mathrm{~mA}$. The diode leads are clad in thin ceramic tubes which insulate the diode from the plasma to minimize localized electrostatic plasma waves induced by the modulating signal. In free space, the scattering cross section of the antenna is a function of $1 / \lambda$ and center loading resistance [10]. For our situation in vacuum, the center loading resistance would correspond to the dipole antenna terminal resistance of the PIN diode in parallel with the two $10 \mathrm{k} \Omega$ bias resistors and the $50 \Omega$ generator internal impedance. In a magnetized inhomogeneous plasma the problem is more complex. We have chosen an antenna of $3 \mathrm{~cm}$ total length which maximizes the backscattered signal level in the propagating region.

The homodyne detection methods that we have developed are adapted from the systems described by King [10] and used by him primarily to measure microwave antenna patterns in vacuum. We wish to measure the scattering at microwave frequencies from a single small object, in the low frequency noise environment of a bounded plasma.

A schematic diagram of the homodyne system is shown in Fig. 3. The microwave signal source $(1.4$ to $3 \mathrm{GHz})$ is routed through a circulator and a pair of matching stubs to the helical transmitting-receiving antenna that is immersed in the plasma. The desired scattering is from a moveable dipole probe which is modulated from $10 \mathrm{kHz}$ to $500 \mathrm{kHz}$ through a microwave or PIN diode centered in the dipole antenna. The low frequency modulation differentiates the scattered signal received from the dipole antenna from the microwave scattering from the rest of the plasma column, other probes, and the plasma source. The scattered signal is received by the same helical antenna and is fed back through the circulator to a balanced mixer. This mixer is also fed a reference signal from the microwave signal source and the output contains the modulated backscatter signal information.

The mixer output is measured with the lock-in voltmeter which has a reference low frequency signal fed to a mixer and acts as a cross correlator and averages the signals over many cycles. The random plasma noise is greatly decreased by the averaging process, producing a phase sensitive voltage that depends on the scattered wave amplitude and phase as $V_{\text {lock-in }} \sim\left|E_{s}\right| \cos \int \bar{k}_{p} \cdot d \bar{s}$ where $\bar{k}_{p}$ is the wavenumber in the plasma. The phase shift information is integrated over the total launched and backscattered spacial trajectory.

To analyze the homodyne system, we assume that the wave launched from the helical antenna is of the form $E_{h} e^{j\left(k_{1} z-\omega t\right)}$. The backscattered signal from the diode antenna is then $E_{d} e^{-j\left(k_{2} z+\omega t\right)}$, where

$$
\begin{gathered}
E_{d} \sim c(t) E_{h} e^{-\gamma_{1}+j \phi_{i}} . \quad \gamma_{1}=\int_{z_{h}}^{z_{d}} \operatorname{Im} k_{1} d z, \phi_{1}=\int_{z_{h}}^{z_{d}} \operatorname{Re} k_{1} d z \\
c(t)=s(t)\left(\sigma_{\max }^{1 / 2}-\sigma_{\min }^{1 / 2}\right)+\sigma_{\min }^{1 / 2}
\end{gathered}
$$

the $\sigma_{\max }$ and $\sigma_{\min }$ are maximum and minimum backscatter cross-section of the diode antenna when it is reverse or forward biased, respectively, and

$$
s(t)=\left\{\begin{aligned}
=1 & \text { for } n T \leq t \leq(n+1 / 2) T \\
=0 & \text { for }(n+1 / 2) T \leq t \leq(n+1) T \\
& \text { where } T=2 \pi / \omega_{m} .
\end{aligned}\right.
$$

The signal at the input of the mixer is proportional to $E_{d} e^{-\gamma_{2}+j \phi_{2}}$ where

$$
\gamma_{2}=-\int_{z_{d}}^{z_{h}} \operatorname{Im}\left(k_{2}\right) d z \text { and } \phi_{2}=-\int_{z_{d}}^{z_{h}} \operatorname{Re}\left(k_{2}\right) d z .
$$

The output of the mixer contains four terms

$$
\begin{aligned}
c_{1}+c_{2}\left|E_{h}\right| e^{-\gamma_{1}-\gamma_{2}} \cos \left(\phi_{1}+\phi_{2}\right) s(t) \\
+c_{3} s(t) \cos \left(2 \omega t+\phi_{1}+\phi_{2}\right)+c_{4} \cos 2 \omega t
\end{aligned}
$$

where the first term represents the DC component, the third and fourth terms are second harmonic components, and the second term is the modulated signal frequency which contains the desired amplitude and phase information of the backscattered signal. The lock-in amplifier output is given by

$V_{\text {lockir } 1}=\frac{C}{2}\left(\sigma_{\max }^{1 / 2}-\sigma_{\min }^{1 / 2}\right)\left|E_{h}\right| \cos \left(\phi_{1}+\phi_{2}\right) e^{-\gamma_{1}-\gamma_{2}}$ where $C$ is a constant.

It is apparent from this expression that in order to increase the detected signal level, one should either increase the launched power or the difference in cross sections when the scatterer is reverse or forward biased. The homodyne system operates with a $20 \mathrm{~V}$ peak-to-peak square wave signal which drives the microwave diode or PIN diode which were used as modulators in the dipole antenna. The dipole antenna, shown in Fig. 2 together with the Langmuir probe, has a $3 \mathrm{~cm}$ total length which extends from the diode with 10 $\mathrm{k} \Omega$ resistors located on each of the feeds to the diode to 


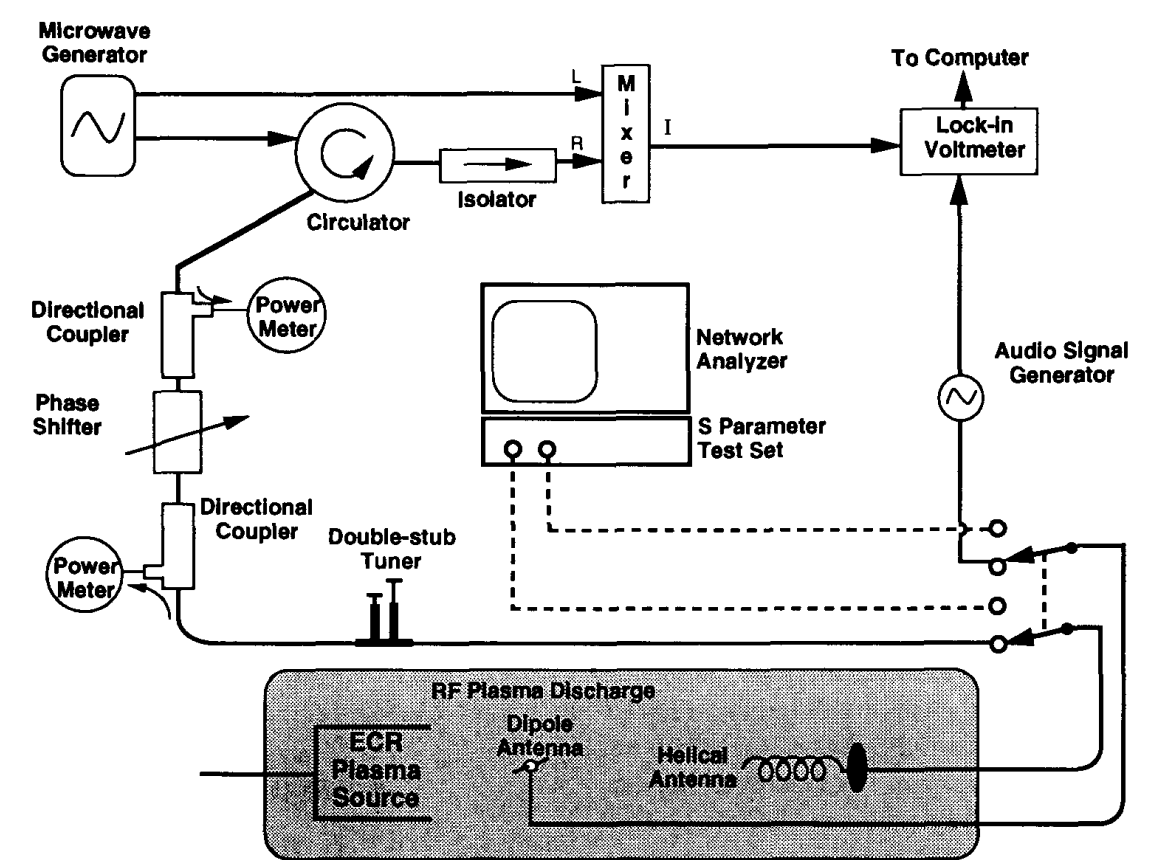

Fig. 3. Schematic of homodyne detection system. The connections for the network analyzer measurements are also shown.

suppress microwave power from being fed back to the coaxial modulating system. This modulation scheme essentially acts as a microwave switch which modulates the microwave diode between a forward bias state in which the resistance seen by the microwaves is very low $(<1 \Omega)$ to the reverse bias state in which the microwave diode has a high resistance $(>1 \mathrm{M} \Omega$ ).

The linear range of the overall homodyne system for a 10 $\mathrm{mW}$ generator output level has been measured to be $50 \mathrm{~dB}$ for the case in which a high frequency $500 \mathrm{kHz}$ lock-in amplifier is used and $73 \mathrm{~dB}$ for the case of a $10 \mathrm{kHz}$ lock-in amplifier signal. The minimum detectable helix launched power was 25 $\mathrm{nW}$ for the case of high frequency modulation and $0.3 \mathrm{nW}$ for the case of low frequency modulation. The dipole scattered power level when located in the wave propagating region and received by the helix is typically $40-50 \mathrm{~dB}$ below these values.

\section{EXPERIMENTAL RESULTS}

\section{A. Direct Transmission Spatial Measurements}

We begin with a discussion of direct measurements of the attenuation of the electron cyclotron waves near cyclotron resonance, in the frequency range of 1.6-2.4 GHz. These measurements differ from those of an earlier paper [6] in two respects: The plasma density was above the threshold density for the electron cyclotron mode $\left(f_{p e}>f\right.$ or $n>5.1 \times 10^{10}$ $\mathrm{cm}^{-3}$ for $f=2 \mathrm{GHz}$ ), and the microwave power was kept low enough so that nonlinear effects were not observed $(P \lesssim 1 \mathrm{~W})$.

The electron cyclotron wave was launched by a helical antenna and two receiving antennas were used: a short electric dipole antenna and the Langmuir probe. The receiving antenna was moved along the axis, passing through cyclotron resonance for most cases. The input microwave power was produced by the same signal generator used in the backscatter experiments and amplified by a broadband traveling wave tube. The received power level was measured by a spectrum analyzer and sent to the computer by the digital interface. The spectrum analyzer sweeps the spectrum periodically and measures the height of the spectral peak.

The input power level was varied from $1 \mathrm{~W}$ to $1 \mu \mathrm{W}$, with no visible spectral sidebands. A typical result is shown in Fig. 4 , showing received power in $\mathrm{dB}$ as a function of axial antenna position $z$. In this case the input power is $P=1 \mathrm{~W}$, the frequency is $f=2 \mathrm{GHz}$, the on-axis plasma density at $z=0$ is $n=1.5 \times 10^{11} \mathrm{~cm}^{-3}$, and the magnetic coil reference current is $I=309$ A. The wave power decreases by $50 \mathrm{~dB}$ near cyclotron resonance in this case. The fluctuations in the power level are typical of the direct attenuation measurements since the probe motion can affect the plasma on flux tubes which link the plasma source and dipole antenna. Lower power results are similar, showing complete attenuation of the wave past cyclotron resonance down to the instrument noise level. In each case, in the high density regime, there is little evidence of standing wave patterns, a result that implies small reflections and substantial absorption.

\section{B. Network Analyzer Frequency Sweeps at Fixed Spatial Positions}

A network analyzer with a two port S-parameter test set [11] was used to measure the broadband frequency response between the helical and dipole antennas for different plasma densities and magnetic fields. The range of plasma parameters was obtained by varying the power level coupled to the plasma source and the magnetic field. The densities were measured by means of a disk Langmuir probe and phase sensitive 


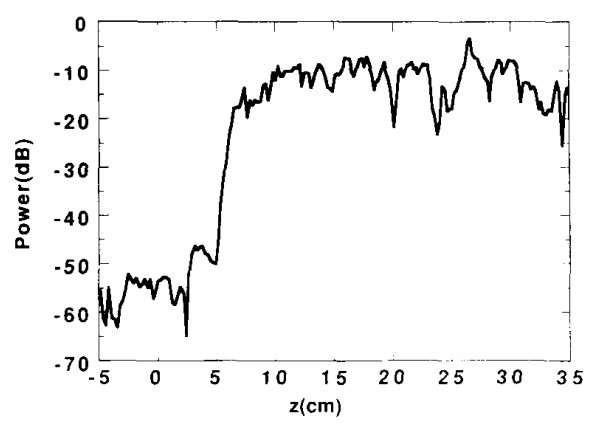

Fig. 4. Received wave power (dB) vs. $z$ for a transmission measurement. Input power $=1 \mathrm{~W}, f=2 \mathrm{GHz}$, plasma density $=1.7 \times 10^{11}$ $\mathrm{cm}^{-3} \cdot I_{c}=309 \mathrm{~A}$.

interferometer measurements of the local wavelengths for the electron cyclotron wave near and somewhat removed from the cyclotron resonance in the inhomogeneous magnetic field.

A comparison of the density measured by Langmuir probes to the plasma density inferred by the theory of parallel propagation in a hot plasma [1], [2] obtained from measurements of local wavelengths near resonance always provided an agreement within $20 \%$. The on-axis density as measured by Langmuir probes was typically lower than that inferred by local wavelength measurements.

A network analyzer trace for a higher density plasma of $3 \times 10^{11} \mathrm{~cm}^{-3}$ at $z=0$ over the frequency range of 13 $\mathrm{GHz}$ is shown in Figs. 5(a) and (b). This was obtained by increasing the plasma source microwave power to $230 \mathrm{~W}$ and magnetic field corresponding to a coil current of $I_{c}=290$ A. The traces are an average of 16 measurements sequenced in rapid succession by the network analyzer source at $3 \mathrm{~mW}$ incident power levels. Fig. 5(a) shows the two port power scattering parameter $\mathrm{S}_{12}$ between that launched by the dipole probe located at $z=30 \mathrm{~cm}$ (top trace) and $z=0 \mathrm{~cm}$ (lower trace) and that received by the helix. Above a frequency of $1.6 \mathrm{GHz}$ the transmitted power between the two antennas is reduced by $20-30 \mathrm{~dB}$ due to the presence of a cyclotron resonance zone which absorbs the launched power between the dipole and helix in the inhomogeneous magnetic field (refer to Fig. 1 for the relative change in the magnetic field versus the $z$ position.).

The scattering element $S_{21}$ is obtained by sending the launched power through the helix to be received by the dipole probe. The result is shown in Fig. 5(b) where the dipole probe is located in the same positions as in Fig. 5(a). In this case the received power is reduced by $50-30 \mathrm{~dB}$ over most of the frequency range where a cyclotron resonant zone lies between the two antennas. The arrow shows the positions of the cyclotron resonance corresponding to the two locations of the dipole antenna. Similar results were obtained when a Langmuir probe tip was substituted for the dipole antenna but the dipole antenna case is emphasized in order to compare with backscatter measurements which utilize the homodyne microwave system.

It is observed that the anisotropic plasma exhibits a nonreciprocal feature which is strongest at higher densities. Figs. 6(a) and (b) show a somewhat lower and uniform density

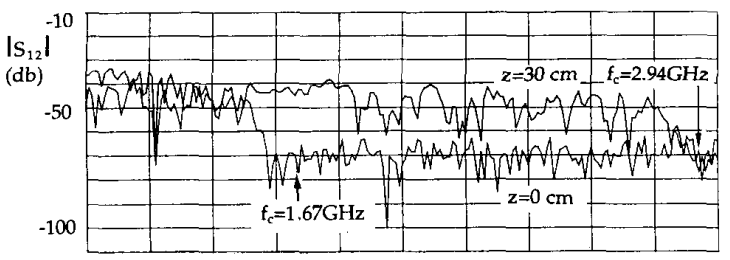

(a)

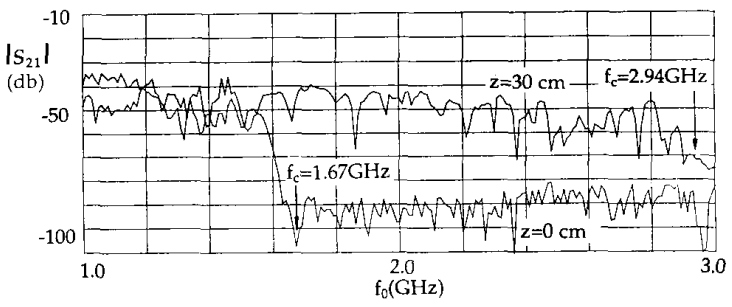

(b)

Fig. 5. Power scattering parameters vs. frequency for the dipole antenna located at two fixed positions: $z=30 \mathrm{~cm}$ (upper trace) and $z=0 \mathrm{~cm}$ (lower trace). a) $\left|S_{12}\right|$; b) $\left|S_{21}\right|$. The plasma density is $3.3 \times 10^{11} \mathrm{~cm}^{-3}$ at $z=0$.

along the axis of $1.5 \times 10^{11} \mathrm{~cm}^{-3}$ which was produced by reducing the magnetron power and changing the coil current to $I_{c}=301 \mathrm{~A}$. In this case the power received is reduced by a similar amount as in the previous case for frequencies just above the cyclotron resonance at the probe position, but for the higher frequency range of the spectrum $(2.3-3.0 \mathrm{GHz})$ an increase of $10-20 \mathrm{~dB}$ in the received power is noted. When even lower density plasmas $\left(n=5 \times 10^{10} \mathrm{~cm}^{-3}\right)$ were created, the two scattering parameters showed a much lower reduction due to the presence of the cyclotron resonance located between the antennas and much more reciprocal features. These results were quite sensitive to the plasma density and its profile. These results will be explained in the discussion section in terms of the allowed launch and received wave modes for the antennas which lie in different regions of the Clemmow-Mullaly-Allis (CMA) diagram

\section{Backscatter Measurements}

We find the scattering measurements made by the homodyne system are very sensitive and reproducible. The method and its linearity ( $70 \mathrm{~dB}$ range) has been checked down to a launched power level of $-70 \mathrm{dBm}$. When the scattering probe tip is modulated at $50 \mathrm{kHz}$ and placed beyond the spatial position corresponding to cyclotron resonance for a high density plasma $\left(n_{e}=3 \times 10^{11} \mathrm{~cm}^{-3}\right.$ measured on-axis at $z=7 \mathrm{~cm}$ ) and a coil current of $I_{c}=310 \mathrm{~A}$, the backscattered $2 \mathrm{GHz}$ signal decreases by $40 \mathrm{~dB}$ as shown in Fig. 7(a). Fig. 7(b) shows an expanded trace of the backscattered signal near and beyond cyclotron resonance. The residual signal in this case is $40 \mathrm{~dB}$ below that in the propagating region. The peak amplitude of the backscattered signal is fairly constant in this case until it decays near $f / f_{c}=0.94$, implying that the transmission path betwen the helix and dipole is dominated by the direct flow of energy axially along the plasma column. The homodyne backscattered signal also shows twice the phase shift due to 


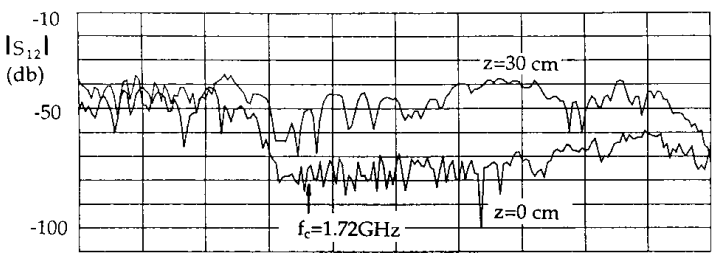

(a)

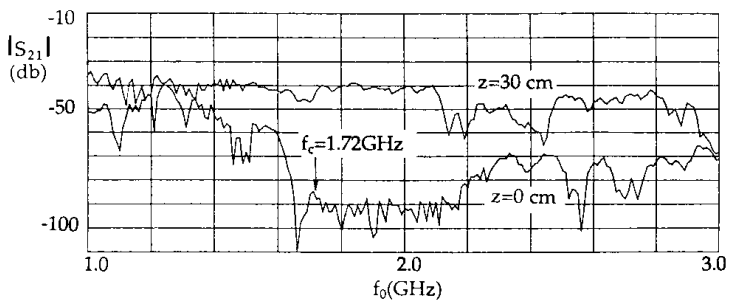

(b)

Fig. 6. Scattering parameters vs. frequency for a plasma density of $1.5 \times 10^{11} \mathrm{~cm}^{-3}$ at $z=0$. The other parameters are the same as in Fig. 5 .

probe motion when compared to the single pass transmission signal phase which is provided by the output of a balanced mixer with a microwave reference signal.

\section{Discussion}

\section{A. Ray Tracing and CMA Trajectories}

The interpretation of the results depends on an understanding of the plasma and wave diagnostic systems and the axial and radial profiles of the magnetic field and plasma density. The behavior of the wave propagation between the helix and the dipole antennas can be interpreted near the axis by ray tracing. This method is also used to indicate the group velocity trajectory and phase velocity direction for rays which propagate significantly off axis to illustrate the energy guiding and absorption properties of the wave in the typical hollow profile plasma column.

The density profile was measured with a $2.4 \mathrm{~mm}$ disk Langmuir probe as a function of radius and axial position. The density model shown in Fig. 8 is used in the ray tracing code UWRay [12] to approximate the measured density profile. UWRay is a reimplementation of the theory presented in [13]. The parameter $\left(f_{p} / f\right)^{2}$ is the square of the ratio of the electron plasma frequency to the wave frequency which is proportional to the plasma density. The corresponding electron temperature profile is much flatter than density with temperatures in the $4 \mathrm{eV}$ range. The 2-D magnetic field was also measured by a Gaussmeter probe and stored for use in ray tracing analysis.

Fig. 9 shows ray trajectories for a $2 \mathrm{GHz}$ wave which is right-hand polarized with respect to the magnetic field and is launched with initial wavevectors oriented 2,4 , and $16^{\circ}$ off the magnetic field axis from the axial position of the helix at the radial center of the plasma. The dotted path is the group velocity trajectory which remains near the center of the plasma and the magnitude and direction of the vectors correspond to

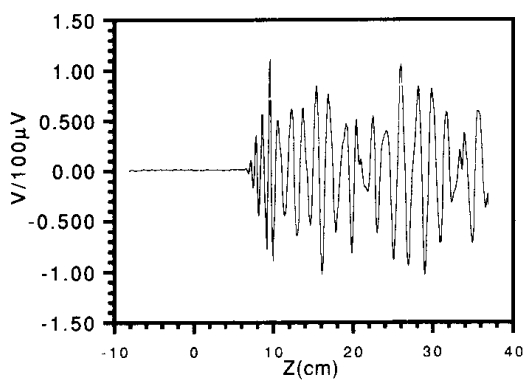

(a)

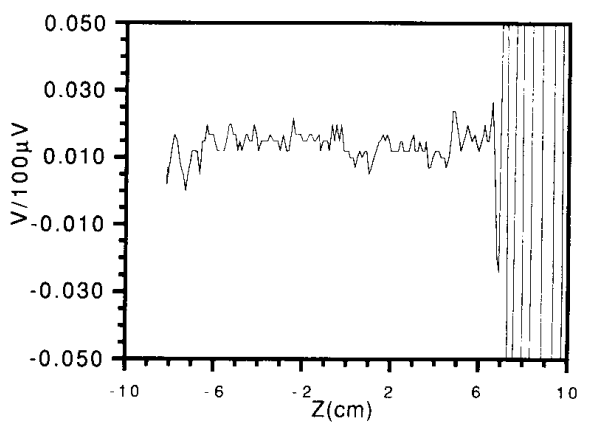

(b)

Fig. 7. Homodyne measured backscatter signal from dipole antenna at $f=2.00 \mathrm{GHz}$. a) Lockin sensitivity at $100 \mu \mathrm{V}$ level. b) Lockin sensitivity increased to $1 \mu \mathrm{V}$ level.

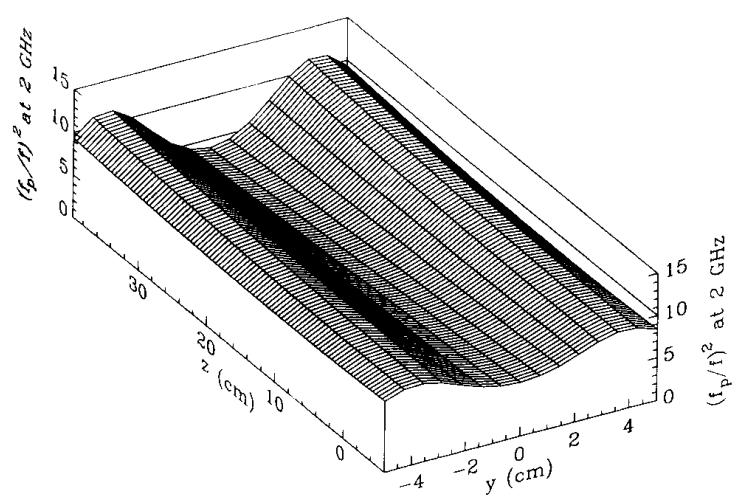

Fig. 8. Smoothed plasma density profile vs. radius $r$ and axial position $z$.

the magnitude and direction of the wavevector which remains nearly parallel to the magnetic field. The figure illustrates that the wave energy flow in the off-axis peaked density profile remains well confined in the plasma core. A check of the ratio of $L_{\min / \lambda}$ along the ray trajectory for the $2^{\circ}$ and $4^{\circ}$ launch angles, where $\lambda$ is the local wavelength and $\mathrm{L}_{\min }$ is the smallest of the gradient scale lengths of density, magnetic field and magnitude of the wavevector evaluated locally along the ray path, shows that $L_{\min } / \lambda$ remains greater than four over the ray trajectory until the cyclotron resonance zone is reached. Although for the $16^{\circ}$ launch angle $L_{\min } / \lambda \gtrsim 1$, the calculated trajectory is still of interest as an indication of the likely qualitative character of the energy flow trajectory. 


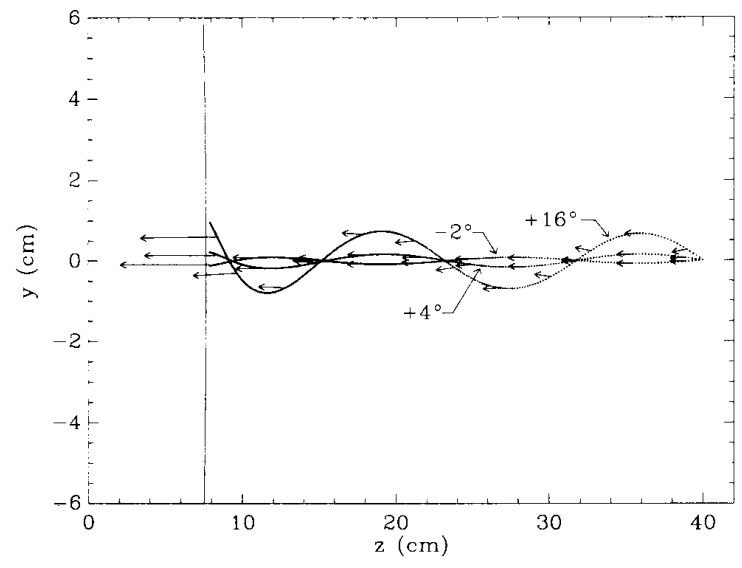

Fig. 9. Ray trajectories obtained using UW-RAY with initial off-axis launching angles of $2^{\circ}, 4^{\circ}$, and $16^{\circ}$.

Several radial measurements of the wave propagation and absorption indicate wave energy flow over the region inside the peaks in the radial density profile with substantial wave absorption near the axial position corresponding to cyclotron resonance. This is comparable to the results suggested by ray tracing.

To further interpret our network analyzer results and the nonreciprocity observed between the two antennas at higher densities, we present the CMA diagram for our plasma in Fig. 10. We interpret the CMA diagram with the usual cold plasma cutoffs and resonances [14] together with a substantial hot plasma cyclotron damping process near the $R=\infty$ resonance. The density as measured by the Langmuir probe is $3.3 \times 10^{11} \mathrm{~cm}^{-3}$ with an axial profile as shown in Fig. 8 as one moves from the local mirror minimum at $z=0$ to the helix antenna. The higher density plasma parameters correspond to those for the network analyzer scattering parameters presented in Figs. 5(a) and (b) and the mode wavenormal surfaces for each of the eight regions are illustrated. The on-axis trajectories from $z=45 \mathrm{~cm}$ at the axial center of the helix to the position $z=0$ at the local mirror minimum are shown from $3-1.6 \mathrm{GHz}$ with a $200 \mathrm{MHz}$ difference between each trajectory. Note that in this case all trajectories lie in the region where the R-mode can propagate and the L-mode is evanescent or in the region below the cyclotron resonance $R=\infty$ where both modes are cutoff. When the dipole receiver probe is located at $z=0$, frequencies above $1.55 \mathrm{GHz}$ are substantially absorbed or have a region between the two antennas where a cyclotron resonance exists. The launch of a pure R-mode, presence of the absorption region, and evanescence above the cyclotron frequency in the CMA diagram shows why the network analyzer trace (Fig. 5(b)) has a 40-50 dB drop at higher frequencies, limited by the noise level of the instrument.

Fig. 11 shows the CMA diagram and frequency trajectories for the case in which the density is lowered to $1.7 \times 10^{11} \mathrm{~cm}^{-3}$. The density is uniform from the magnetic field minimum to the helix position at $z=45 \mathrm{~cm}$. Note from the corresponding network analyzer trace on Fig. 6(b) that the damping of the $S_{21}$

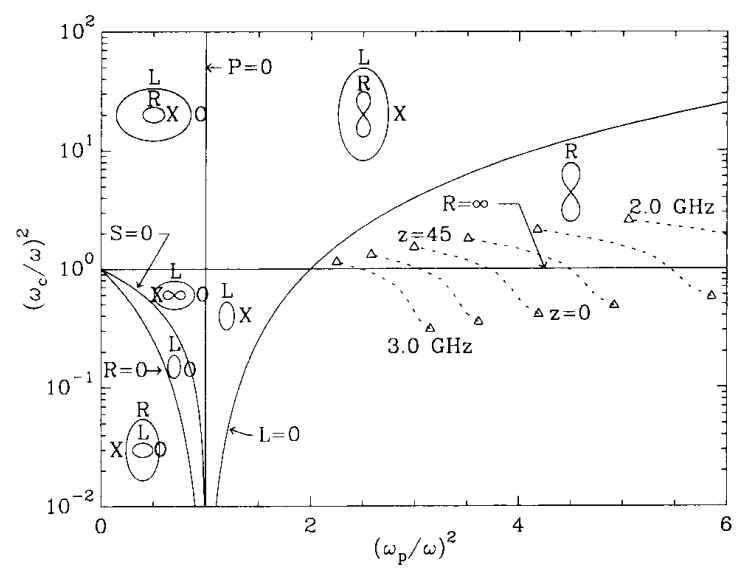

Fig. 10. CMA diagram and wave trajectories for a higher density plasma (3.3 to $1.7 \times 10^{11} \mathrm{~cm}^{-3}$ ). Refer to Figs. 5(a) and (b).

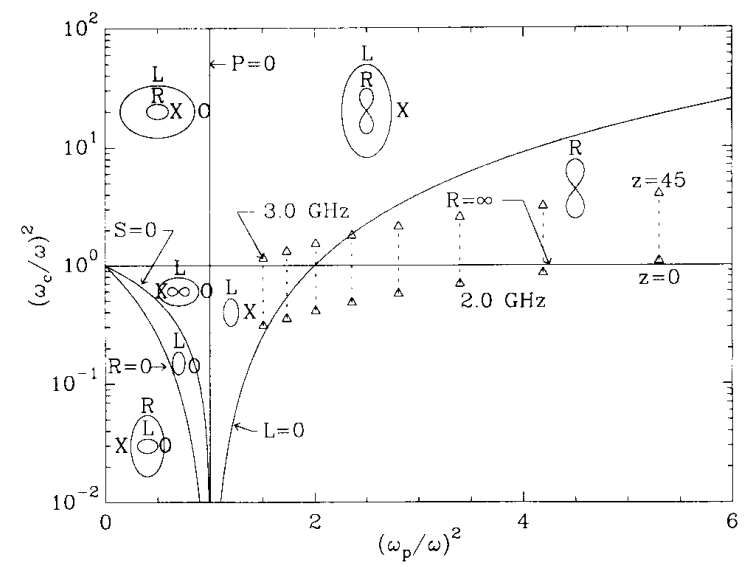

Fig. 11. CMA diagram and wave trajectories for a lower density plasma $\left(1.7 \times 10^{11} \mathrm{~cm}^{-3}\right)$. Refer to Figs. 6(a) and (b).

frequency components above $2.3 \mathrm{GHz}$ is smaller for this case than for the higher density regime. This is because the higher frequency components lie above the $L=0$ surface in the CMA diagram and both the $\mathrm{L}$ - and $\mathrm{R}$-modes can propagate in the vicinity of the helix launch antenna. The antenna launches some residual L-mode characteristic at this lower density which is not absorbed near the $R=\infty$ cyclotron resonance.

\section{B. Nonreciprocal Features of the Scattering Elements}

For the scattering matrix element $\mathrm{S}_{12}$, the dipole antenna launches the waves and they are received by the helical antenna. For the trace $S_{21}$, the helix primarily excites the R-mode propagating opposite to the direction of the magnetic field. At lower plasma densities $\left(n=5 \times 10^{10} \mathrm{~cm}^{-3}\right)$, the transfer scattering matrix elements for the two antennas are reciprocal. As the plasma density is increased, more marked differences occur as illustrated in Figs. 5(a) and 5(b) for frequencies above the region corresponding to cyclotron resonance at the 
launching antenna. The propagating frequencies show a nearly reciprocal characteristic for these two cases as well as all other cases which were examined. However, for damped and cutoff frequencies at high density, $\mathrm{S}_{12}$ is about $20 \mathrm{~dB}$ above the case for $S_{21}$. A plausible explanation for these differences involves the polarization characteristics of the two antennas, mode propagation in different regions of the CMA diagram, and absorption processes in the plasma.

For frequencies above $1.6 \mathrm{GHz}$ in the $\mathrm{S}_{12}$ trace of Fig. 5(a) the dipole antenna is located in the lower right part of the CMA diagram (Fig. 10), so both the $\mathrm{R}$ and $\mathrm{L}$ mode excitations are evanescent. The linear dipole antenna excites the $\mathrm{R}$ - and L-mode polarization about equally and with a much broader range of $k$-vector angles to the magnetic field than the helix. The left-hand polarized portion of this wave energy which is substantial even for the R-mode at large angles relative to the magnetic field, can pass through the electron cyclotron resonance zone unaffected; therefore, a smaller percentage of the wave energy excited by the dipole is lost through absorption in the cyclotron resonance zone than for the energy launched by the helix for the $S_{21}$ case. The wave energy which passes through the resonance zone from the dipole is transmitted to the helix via large angle scattering off the plasma edge region and/or mode conversion of the L-mode energy into the R-mode.

\section{Backscatter Measurements}

The peak amplitude of the backscattered signal is fairly constant for Fig. 7(a) until it swells and then decays near $f / f_{c}=0.94$ implying that the transmission path between the helix and dipole is dominated by the direct flow of energy axially along the plasma column. We often observe that wave swelling occurs just preceding the absorption zone and that strong wave absorption occurs at slightly higher magnetic field strengths than would be predicted by WKB hot plasma theory for our electron temperatures and densities. This observation is in agreement with the kinetic full wave theory of Fruchtman et al. [15] which predicts initial wave swelling and a strong wave absorption shifted to lower values of $f / f_{c}$.

\section{Comparison of Spatial Damping and Wavenumber Measurements with WKB and Bounded Plasma Theory}

The backscatter and network analyzer data are used to provide measurements of local wavenumber $\left(k_{r}\right)$ and collisionless cyclotron damping decrements $\left(k_{i}\right)$ for the electron cyclotron wave in the inhomogeneous local mirror magnetic field. The real part of the local wavenumber is obtained from a fast Fourier transform analysis of the backscattering results shown in Fig. 7(a) done at $f=2.00 \mathrm{GHz}$ at a radius of $2 \mathrm{~cm}$. The backscatter trace is windowed in selected spatial zones of the phase sensitive backscatter signal. A spatial fast Fourier transform analysis is applied for each case to obtain the dominant wavenumber in the spectrum for the average spacial position in the window. Since the backscatter signal involves a round trip from the helix to the dipole scatterer and return, the backscatter

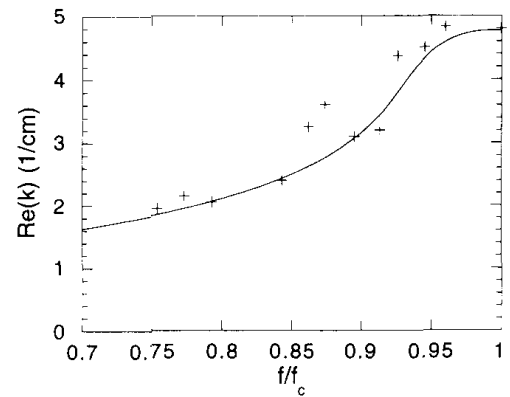

(a)

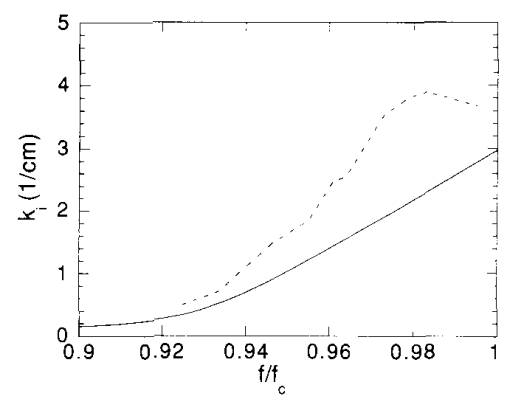

(b)

Fig. 12. Quantitative comparison of experimental and WKB theoretical results. (a) Local wave numbers obtained from $f=2.00 \mathrm{GHz}$ backscatter measurement (crosses, solid line corresponds to hot plasma theory). (b) Wave damping obtained from network analyzer measurements (dashed line, $f_{c}=1.73 \mathrm{GHz} \mathbf{\alpha} z=3.25 \mathrm{~cm}$; solid line, corresponding hot plasma theoretical results).

$k$-spectrum value is divided by a factor of two to determine the local wavelength at the average window position.

The network analyzer transmission power scattering coefficient results are a function of both frequency and the location of the dipole receiver. By moving the dipole receiver a small distance, $\Delta z=0.5 \mathrm{~cm}$, and noting the change in received power level at different frequencies, we can determine the average damping decrement over that spacial distance as a function of frequency. We assume that the wave power varies near cyclotron resonance as $\exp \left(-2 \int k_{i} d z\right)$. Then the average damping decrement over the distance $\Delta z$ is given by the expression

$k_{i}($ Nepers $/ \mathrm{cm})=\Delta S_{21}(\mathrm{~dB})_{f} / \Delta z(\mathrm{~cm}) / 8.686(\mathrm{~dB} /$ Neper $)$.

Fig. 12(a) shows a comparison between the local hot plasma theoretical [1], [2] and experimentally obtained wave number values for a backscatter wave frequency of $f=2.00 \mathrm{GHz}$. The plasma parameters obtained for the experiments from Langmuir probe data correspond to a density of $n=3 \times$ $10^{11} \mathrm{~cm}^{3}$ and an electron temperature of $T_{e}=3.5 \mathrm{eV}$.

We note that the measured wave propagation phase shift and absorption are in close agreement with a quasi-parallel, near perfect polarization in the sense of electron rotation about the magnetic field yielding $40-50 \mathrm{~dB}$ attenuation through cyclotron resonance. This is particularly noteworthy since the 
column is $12 \mathrm{~cm}$ in diameter near the electron cyclotron resonance zone where $f / f_{c e}=0.9$. One could ask how a finite column could allow wave propagation close to that for an unbounded plasma for this case. To address this we compute the normal mode characteristics for a uniform, magnetized hot plasma column with a vacuum boundary condition at the plasma edge radius $r=6 \mathrm{~cm}$. This problem has been addressed for exmaple in the limit of a cold plasma in the text by Swanson [16]. The problem of wave propagation in a hot Maxwellian cylindrical plasma has been analyzed by McVey who has written a computer code called ANTENA [17] which has been used for many problems of this sort including ion cyclotron frequency range wave propagation and heating for mirror machines including TARA [18].

The ANTENA code calculates the plasma-wave fields generated by a set of coils external to a cylindrical plasma. We have applied this code to a calculation of the plasma fields generated by a Nagoya type III coil in a cylindrical plasma with density, temperature and $k_{z}$ values comparable to values measured in our experiment at $f / f_{c e}=0.9$. In particular, the code can isolate the mode with azimuthal number $m=1$ and compute its propagation characteristics. The results show that such a mode with the proper experimental parameters $\left(k_{z}=\right.$ $\left.3.2 \mathrm{~cm}^{-1}, E_{r} / E_{l}=10^{+3}, k_{\perp} \ll k_{\|}\right)$can be maintained inside the plasma.

To illustrate our case, we assume $f / f_{c e}=0.9, f=2 \mathrm{GHz}$, $\left(f_{p} / f\right)^{2}=6, T_{e}=5 \mathrm{eV}$, a plasma radius of $r=6 \mathrm{~cm}$ and a conducting boundary at $r=8 \mathrm{~cm}$. We find from the ANTENA code that the dominant $m=1$ azimuthal normal mode in the plasma excited by the helix that an electron cyclotron wave with $k_{z}=3.2 \mathrm{~cm}^{-1}, k_{\perp}=0.42 \mathrm{~cm}^{-1}$ exists. We further find that the total wave field polarization is dominantly in the sense of electron rotation about the magnetic field with $\left|E_{r} / E_{l}\right|>100$ over the inner two thirds of the plasma radius. The $m=0$ and $m \geq 2$ modes are only weakly excited by the helix in the plasma column. Thus we find from our computational studies that a finite radius, vacuum boundary plasma column can support quasi-parallel wave propagation and energy flow when the wave frequency is reasonably close to cyclotron resonance, in agreement with our ray tracing results and experimental measurements.

The experimental damping decrement, $k_{i}$, is obtained as a function of frequency from the difference in the network analyzer transmission scattering parameter traces at $z=3.0$ and $z=3.5 \mathrm{~cm}$. This corresponds to an average spacial position of $3.25 \mathrm{~cm}$ and a cyclotron resonance frequency at the dipole of $1.73 \mathrm{GHz}$. The damping decrement is shown by the dashed line on Fig. 12(b). Note that the measured damping decrements near cyclotron resonance are larger than predicted by WKB theory [1], [2], shown by the solid line on the figure and that significant damping decrements are shifted further away from the cold electron cyclotron resonance. This phenomenon has been discussed in theoretical work by Fruchtman et al. [15] where the WKB and full wave energy flux profiles are compared for plasmas in which WKB criteria are not satisfied due to strong damping. This experimental result corresponds to the theoretical arguments for a more rapid decrease in energy flux in a strong damping region discussed in their paper. Note that the experimental values of $k_{i} / k_{r}$ are of order $0.3-0.6$ over a wide range near cyclotron resonance, thus clearly violating the WKB wave condition, $\left|k_{i} / k_{r}\right| \ll 1$. In addition, the magnetic field gradient length is $L_{B}=18 \mathrm{~cm}$ near the resonance position so that the wave rapidly reaches the strong damping region near resonance where WKB theory cannot strictly be applied.

\section{CONCLUSIONS}

We have investigated the propagation, broadband absorption, and scattering of electron cyclotron waves from a dipole antenna in an inhomogeneous plasma. The use of network analyzer and homodyne modulated scatterer techniques have allowed more detailed results to be obtained which describe the properties of waves launched in an inhomogeneous, warm plasma and their transmission between and backscattering from dipole and helical antennas. The use of direct transmission between the helical antenna launcher and a dipole shows that $-50 \mathrm{~dB}$ attenuation of the signal for plasma densities of $3 \times 10^{11} \mathrm{~cm}^{-3}$ and temperatures of $4 \mathrm{eV}$ can be obtained through the cyclotron resonance. This is comparable to the integral of the hot plasma damping decrement through the resonance zone with negligible collisional effects.

The network analyzer results show that transmission losses of $40-50 \mathrm{~dB}$ occur when a cyclotron resonance is located between the helical antenna launcher and dipole antenna receiver. This can be obtained over a frequency range of $1.5-3.0 \mathrm{GHz}$ for the plasma parameters observed in the inhomogeneous magnetic field. The results show a doppler shifted absorption which starts at $f / f_{c}=0.92--0.94$ which is slightly before that predicted by WKB hot plasma cyclotron damping and in closer agreement with a full wave kinetic theory [15]. It is concluded that the plasma column and helical antenna launch parameters admit only very pure $(-40 \mathrm{~dB})$ right-hand polarized modes to be received by the dipole antenna.

The two antenna system shows a nonreciprocal behavior at higher densities due to the hot plasma. The difference in the two directions for transmission signals at frequencies where absorption and evanescence zones exist between the two antennas is $20 \mathrm{~dB}$ at higher plasma densities and is reduced as the plasma density is decreased. It is argued that this is due to the difference in launched power characteristics for the two antennas and the consequent mode conversion for evanescent waves in a hot, anisotropic plasma as wave power propagates between them.

The modulated scatterer and homodyne system is used to determine the microwave backscatter from a given region of the plasma where all other reffections in a finite plasma column are suppressed. Reductions in the backscatter of 40 $\mathrm{dB}$ are observed due to the presence of a cyclotron resonance zone between the two antennas. This compares with $30 \mathrm{~dB}$ microwave backscatter reductions observed by Destler et al. [19] for pulsed plasmas produced by coaxial guns in the absence of a magnetic field. Backscatter wavelengths half the local hot plasma cyclotron wavelength are also observed for our case. Fast Fourier transform analysis of the real part of the 
backscatter signal is shown to agree well with quasi-parallel propagation hot plasma theoretical predictions for local wave numbers. This method is shown to be sensitive over a 70 $\mathrm{dB}$ dynamic range and down to launched power levels of $-70 \mathrm{dBm}$. Ray tracing results in an inhomogeneous plasma column indicate quasi-parallel energy flow near the center of the plasma column. In addition, a vacuum boundary analysis of a plasma column and use of the ANTENA code is shown to yield quasi-parallel $\left(k_{z} \gg k_{\perp}\right)$ propagation for electron cyclotron waves near cyclotron resonance $\left(f / f_{c e} \simeq 0.9\right)$ for our plasma parameters.

Quantitative analysis of the broadband network analyzer transmission and backscatter measurements were carried out. Near the position of cyclotron resonance, the measured damping decrement increases above that predicted by local WKB hot plasma collisionless damping, in agreement with the strong damping predicted by Fructman et al. [15].

\section{ACKNOWLEDGMENT}

The authors would like to thank Joe Burke, Mike Klabunde, and Dimitri Synitsyn for technical assistance.

\section{REFERENCES}

[1] B. McVey and J. Scharer, "Measurements of collisionless electron cyclotron damping," Phys. Rev. Lett., vol. 31, p. 14, 1973

[2] B. D McVey and J. E. Scharer, "Experimental verification of collisionless electron cyclotron damping," Phys. Fluids, vol. 17, p. 142, 1974.

[3] T. L. Owens and J. E. Scharer, "Thermal effects on electron cyclotron resonance heating." Plasma Phys., vol. 18, p. 663, 1976.

[4] J. E. Scharer and J. E. Mitzlaff, "Excitation of parametric instabilities by electron cyclotron waves in a plasma column," Plasma Phys, vol. 19. p. $413,1977$.

[5] B. W. Rice and J. E. Scharer, "Observation of localized clectron cyclotron resonance heating in a magnetic mirror," IEEE Trans. Plasma Sci., vol. 14, p. 17, 1986.

[6] R. R. Mett, S. W. Lam, and J. E. Scharer. "Experimental investigation of a localized electron temperature clevation," IEEE Trans. Plasma Sci., vol. 17, p. 818,1989

[7] J. H. Booske, W. D. Getty, R. M. Gilgenbach, and R. A. Jong, "Experiments on Whistler mode electron-cyclotron resonance plasma startup and heating in an axisymmetric magnetic mirror," Phys. Fluids, vol. 28, p. 3116,1985

[8] R. L. Stenzel and J. M. Urrutia, "Force-free electromagnetic pulses in a laboratory plasma," Phys. Rev. Lett., vol. 65, p. 2011, 1990.

[9] W. Stutzman and G. Theile, Antenna Theory and Design. New York: John Wiley d Sons, 1981.

[10] R. J. King, Microwave Homodyne Systems. England: Peter Peregrinus Ltd., 1978.

[11] D. M. Pozar, Microwave Engineering. Reading, MA: Addison Wesley, 1990.

[12] M. Bettenhausen and J. E. Scharer, "A ray tracing study of electron cyclotron waves in a bounded plasma experiment," Bull. of the American Physical Society, vol. 35, p. 2066, 1990.

[13] N. T. Lam, J. E. Scharer, and K., R. Audenaerde, "Electron cyclotron resonance heating in tandem mirrors," IEEE Trans. Plasma Sci., vol. 13, p. $25,1985$.

[14] T. Stix, Waves in Plasmas. New York: American Institute of Physics, 1992.

[15] A. Fruchtman. K. Reidel, H. Weitzner, and D. B. Batchelor, "A kinetic full wave theory of strong spatial damping of electron cyclotron waves in nearly parallel stratified plasmas," Phys. Fluids, vol. 30, p. 115, 1987.

[16] G. Swanson, Plasma Waves. San Diego, CA: Academic Press, 1989.

[17] B. D. McVey, "ICRF antenna coupling theory for a cylindrically stratified plasma," M.I.T. Plasma Fusion Center, Report PFC/RR-84-12, 1984.
[18] S. N. Golovato et al., "Plasma production and heating in a tandem mirror central cell by radio-frequency waves in the ion cyclotron frequency range," Phys. Fluids, vol. 31, p. 3744, 1988.

[19] W. W. Destler, J. E. DeGrange, H. H. Fleishmann, J. Rodgers, and Z. Segalov, "Experimental studies of high-power microwave reflection, transmission, and absorption from a plasma-covered plane conducting boundary," J. App. Phys., vol. 69, p. 6313, 1991.

John E. Scharer (M'90) received the B.S., M.S. and Ph.D. degrees in electrical engineering from the University of California, Berkeley, in plasma physics.

$\mathrm{He}$ is a Professor with the Department of Electrical and Computer Engineering, University of Wisconsin, Madison, He has spent research sabbaticals at the CEA Fontenay-aux-Roses, France, in 1970 and at the JET tokamak at Culham, England in 1983. He has been active with graduate students and scientists in theoretical, computational, and experimental research in the area of linear and nonlinear plasma waves and heating of fusion plasmas and lasers. He also teaches and carries out research in the areas of free-electron lasers and Cerenkov amplifiers.

Dr. Scharer is a member of the IEEE, Tau Beta Pi, the American Physical Society, and the Center for Plasma Theory and Computation at the University of Wisconsin.

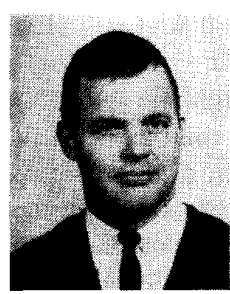

Owen C. Eldridge was born in Crossville, TN, in 1932. He received the B.S. degree in engineering physics from the University of Tennessee, Knoxville, in 1953, and the Ph.D. degree in physics from the University of California, Berkeley, in 1960.

$\mathrm{He}$ has done both theoretical and experimental research at General Atomics in San Diego and at Oak Ridge National Laboratory, concentrating on computer simulations of plasmas, nonlinear instabilities and waves in plasmas, microwave scattering. the theory of the free electron laser, and in the RF heating of plasmas. He has taught physics and engineering at the University of Tennessee, Knoxville, at the City University of New York, Hunter College, and at the University of Wisconsin, Madison.

Dr. Eldridge is a member of Tau Beta Pi and the American Physical Society.

Sheng-Fuh R. Chang (S'83-M'84-S'91-M'91) was born in Taiwan, Republic of China, in 1960 . He received the B.S. and M.S. degrees in communication engineering from the National Chiao-Tung University in 1982 and 1984, respectively. He received the Ph.D. degree in electrical and computer engineering in 1991 and was a member of the Center for Plasma Theory and Computation at the University of Wisconsin, Madison.

His research interests are in theoretical and computational investigations in the area of coherent radiation sources, plasma waves, and microstrip circuits. he currently works for the HYTON Corporation in the area of microwave satellite circuits in Hsinchu, Taiwan.

Mr. Chang is a member of Phi Tau Phi and Sigma Xi.

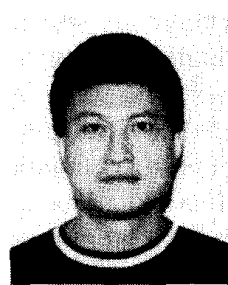

Yong-Shan Zhang received the M.S. degree in electrophysics and Ph.D. degree in electrical engineering from the Polytechnic University, Brooklyn, New York, in 1988 and 1991, respectively. He received his undergraduate diploma in electrical engineering from Nanjing Institute of Telecommunications, Nanjing, People's Republic of China. His graduate research topics included experimental study of high power microwave pulse. 


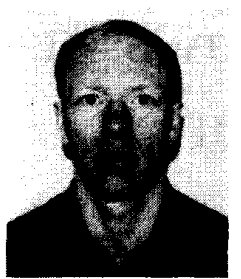

Michael H. Bettenhausen received the B.S. and M.S. degrees in electrical engineering from the University of Wisconsin-Madison in 1983 and 1990 , respectively.

From 1983 through 1987 he served in the U.S. Navy as a nuclear engineer at the Naval Sea Systems Command in Arlington, Virginia. He is currently studying for the Ph.D. in Electrical Engineering at the University of Wisconsin-Madison. His research interests include theoretical and computational studies of radio frequency heating in plasmas. He is currently modeling coupling of ion cyclotron range of frequency waves into tokamak plasmas for plasma heating and non-inductive current drive experiments.

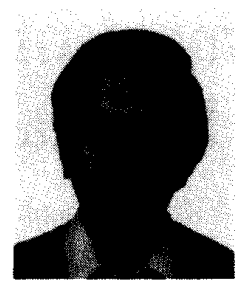

Nguyen T. Lam received the B.S. degree in engineering physics from the University of Montreal in 1969, the M.S. degree in physics from the University of Waterloo in 1971, the Ph.D. degree in physics from Michigan State University in 1977, and the M.S. degree in electrical engineering from The University of Wisconsin in 1983 .

$\mathrm{He}$ is currently a researcher in the Center for Plasma Theory and Computation at the University of Wisconsin-Madison. Since 1980, he has carried out research in plasma heating by RF waves in both the Ion and Electron Cyclotron Range of Frequencies. He has published papers dealing with ray tracing in tandem mirrors and waveguide launchers for tokamaks. His recent interests include studies of plasma production by an excimer laser. 